\title{
Population Structure and Disease Development of Cryphonectria parasitica in European Chestnut Forests in the Presence of Natural Hypovirulence
}

\author{
Martin Bissegger, Daniel Rigling, and Ursula Heiniger
}

Department of Forest Ecology, Swiss Federal Institute for Forest, Snow, and Landscape Research, CH-8903 Birmensdorf, Switzerland. Accepted for publication 17 September 1996.

\section{ABSTRACT}

Bissegger, M., Rigling, D., and Heiniger, U. 1997. Population structure and disease development of Cryphonectria parasitica in European chestnut forests in the presence of natural hypovirulence. Phytopathology 87:50-59.

The Cryphonectria parasitica populations in two 6-year-old European chestnut (Castanea sativa) coppices were investigated in southern Switzerland over a period of 4 years. Occurrence of white isolates indicating an infection with Cryphonectria hypovirus, vegetative compatibility groups (VCGs), hypovirulence conversion capacity, and mating types were used to characterize the populations. Sampling of randomly chosen cankers in the first year yielded 59\% white isolates in one and $40 \%$ in the other population. The distribution of the VCGs and mating types was similar among white and orange isolates, indicating a homogeneous infection of the two populations by the hypovirus. Fourteen VCGs were found in the first population, 16 VCGs in the second. Altogether, 21 VCGs were determined. The same three VCGs dominated in both populations, comprising more than $60 \%$ of all isolates. Several VCGs were represented only by white isolates. Five of the six most common VCGs were clustered in two hypovirulence conversion groups, with almost $100 \%$ hypovirus transmission within each cluster. Repeated sampling of the same cankers in 1990, 1992, and 1994 did not reveal an increase of white isolates. The portion of blighted stems rose from $37 \%$ to about $60 \%$ in both plots within 4 years. In this time, chestnut blight killed $15 \%$ and competition an additional $21 \%$ of the sprouts. Predominantly, sprouts with low diameters at breast height were killed. The growth rate of new cankers was high in their first year and decreased gradually in the following years. A role of hypovirulence in the decline of disease severity was evident since (i) cankers yielding white isolates grew slower and killed considerably fewer sprouts than cankers with orange isolates; and (ii) the majority of the cankers yielded white isolates at least once during the 4-year observation period.

Additional keywords: biological control, dsRNA, Endothia parasitica, epidemiology.
The chestnut blight fungus (Cryphonectria parasitica (Murrill) Barr; Endothia parasitica (Murrill) P.J. Anderson \& H.W. Anderson) destroyed most of the American chestnut trees (Castanea dentata (Marsh.) Borkh.) after its introduction into the eastern United States in the beginning of this century (3). When the blight was introduced into Europe, a similar epidemic in the European chestnut (Castanea sativa Mill.) stands was observed. Although heavily infested by the fungus, the European chestnut stands recovered from the introduced disease in many regions. This has mainly been attributed to the natural occurrence of hypovirulent $C$. parasitica strains $(24,30)$.

Hypovirulent $C$. parasitica strains are infected with doublestranded (ds) RNA viruses $(18,20)$ that are located in the cytoplasm of the fungus and have recently been classified as Cryphonectria hypovirus (31). Hypovirulent isolates produce nonlethal, superficial cankers that are restricted to the outer parts of the bark and do not destroy the vascular cambium of the host. Beside hypovirulence, virus-infected $C$. parasitica isolates often show reduced sporulation and pigmentation, resulting in a white appearance when grown in culture $(18,20,21)$.

The hypovirus can be transmitted through hyphal anastomosis, resulting in conversion of the recipient strain to the hypovirulent phenotype $(7,48)$. Transmission of the hypovirus is limited by a vegetative incompatibility system in $C$. parasitica involving five to seven vegetative incompatibility (vic) loci (4). Compatible fungal strains share identical alleles at all vic loci and are assigned to the same vegetative compatibility group (VCG). The hypovirus is

Corresponding author: M. Bissegger; E-mail address: martin.bissegger@wsl.ch

Publication no. P-1996-1105-01R

(C) 1997 The American Phytopathological Society readily transmitted between strains that belong to the same VCG $(1,10,33,47)$. Transfer of the hypovirus can also occur between strains in different VCGs, but more slowly and at lower rates $(7,34,35)$.

Sexual recombination of polymorphic vic loci is thought to be the main source of VCG diversity. Two mating type alleles (MAT1-1 and MAT1-2) at a single locus control sexual compatibility in $C$. parasitica (4). Sexual recombination takes place between strains carrying different alleles at the mating type locus. However, the fungus can also self-fertilize $(4,39)$. Both sexual and asexual reproduction occur in populations of $C$. parasitica $(9,38)$. In a population with predominantly sexual reproduction, a mating type ratio of 1:1 is expected. Sexual reproduction in $C$. parasitica populations has a negative impact on the dissemination of hypovirulence, because the hypovirus is not transmitted into ascospores $(4,17)$. If the mating type ratio is not $1: 1$, then asexual reproduction could play a major role in the population structure and favor the spread of the virus that causes hypovirulence. Thus, the population structure, with special regards to VCGs and mating types, becomes an important factor for the success of hypovirulence in $C$. parasitica populations.

In southern Europe, hypovirulence is naturally disseminated and also artificially applied as a biocontrol agent $(25,49)$. Unfortunately, hypovirulence has not developed naturally in the United States. This has mainly been explained by a greater diversity of VCGs in United States than in European populations of $C$. parasitica (8). Hypovirulent strains containing European hypovirus were artificially introduced into American forest clear-cuts with abundant chestnut regeneration, but were not successful in controlling the developing blight epidemic $(9,37)$. Improved chestnut tree conditions after treatment with hypovirulent strains, however, were reported from a study in Connecticut (5). Outside the natural 
range of Castanea dentata, recovery of American chestnuts from the blight was ascribed to the natural occurrence of hypovirulent strains (22). Current attempts to control chestnut blight also involve genetic engineering of $C$. parasitica to improve biocontrol using hypovirulence (41).

The wide distribution of hypovirulent $C$. parasitica strains in many areas in Europe is not completely understood since (i) hypovirulent isolates generally produce less asexual conidia than virulent isolates, (ii) a considerable amount of the conidia produced by hypovirulent isolates do not yield white culture types of C. parasitica, (iii) sexual ascospores that are responsible for longdistance transport do not carry hypovirulence, and (iv) vegetative incompatibility between $C$. parasitica isolates acts as an obstacle for the transmission of hypovirulence through hyphal anastomoses.

Several small area populations of $C$. parasitica have been investigated in the United States $(6,9,29,34,36,40)$. Comparable studies from Europe in which hypovirulence is successful in many areas (30) are lacking. The widespread occurrence of hypovirulence in southern Switzerland provides an ideal opportunity to investigate this phenomenon in its natural environment.

The purpose of this study was to provide quantitative information about the epidemic of chestnut blight in two young coppices of European chestnuts. First, we investigated the genetic structure of $C$. parasitica in these two plots and assessed the potential for the spread of the Cryphonectria hypovirus within the populations. Culture type, vegetative compatibility, hypovirulence conversion capacity, and mating type were used to characterize the isolates and to analyze their spatial distribution. We assumed that a virus had to homogeneously infect a pathogen population to be effective as a biological control agent. For natural biocontrol of $C$. parasitica, it is expected that all common VCGs in a population are infected by the hypovirus and that the movement of the hypovirus among VCGs is not considerably restricted. Second, we followed the development of chestnut blight in the two plots over a period of 4 years to study the interactions of populations of $C$. parasitica with its host Castanea sativa. In particular, we were interested to investigate the impact of $C$. parasitica on sprout mortality, to determine the role of hypovirulence for the growth of naturally occurring cankers, and to observe the dynamics of virulent and hypovirulent fungal strains over the course of time.

\section{MATERIALS AND METHODS}

Study sites. Two Castanea sativa coppice stands in southern Switzerland had been clear-cut 6 years before the study was started (Fig. 1). The chestnut stands were $5 \mathrm{~km}$ apart and separated by $1 \mathrm{~km}$ of chestnut-free area. At each site, a plot was established approximately $40 \times 40-\mathrm{m}$ square containing either 36 or 40

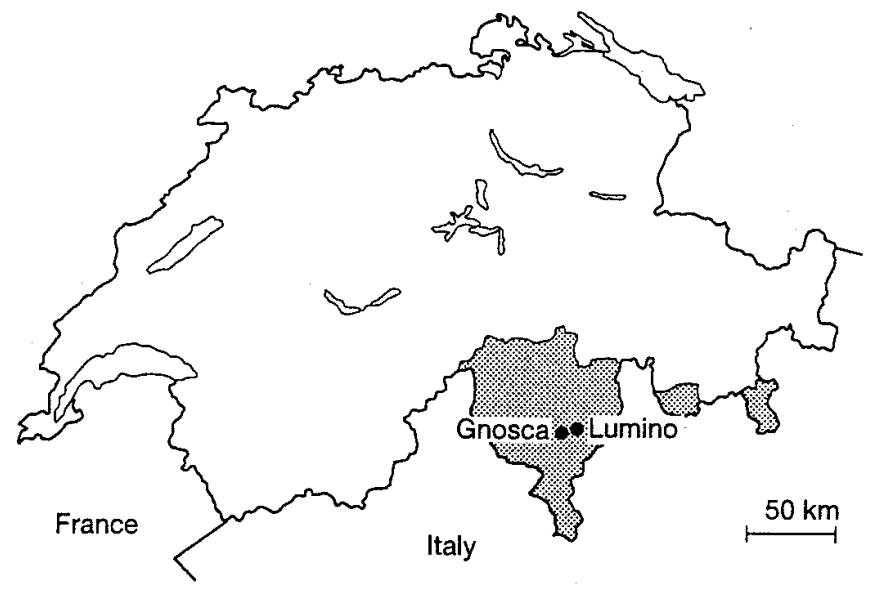

Fig. 1. Location of the two study sites in southern Switzerland. resprouting chestnut stumps (Fig. 2). There were no other competing hardwood species present. The plot Gnosca is located 250 to $280 \mathrm{~m}$ above sea level on a slope (inclination 20 to $60 \%$ ) exposed to the northeast, whereas the plot Lumino is situated 410 to $420 \mathrm{~m}$ above sea level on a slope (inclination 10 to 20\%) exposed to the southeast. Both clear-cuts are surrounded by old grown coppice and mature chestnut tree stands.

Isolation and characterization of $\boldsymbol{C}$. parasitica. In August 1990 , bark samples were removed with a cork borer $(5 \mathrm{~mm}$ in diameter) from the upper and lower margins, as well as from the center of 190 randomly chosen cankers (100 from Lumino and 90 from Gnosca). Within $24 \mathrm{~h}$, all bark samples were dipped in $70 \%$ ethanol, immediately flamed, and placed on $12 \mathrm{~g} /$ liter of malt (Oxoid Division, Oxo Ltd., London) agar supplemented with 0.1 g/liter of Streptothenat (Grünenthal GmbH, Stolberg, Germany). One $C$. parasitica isolate per canker was randomly selected for further analysis.

All cankers that yielded C. parasitica in 1990 were sampled again in August 1992 and August 1994. Cankers on dead sprouts were included in the sampling. In addition, 30 cankers with the highest and 30 cankers with the lowest growth rates between 1990 and 1992 were sampled in August 1992. All C. parasitica isolates were stored on slants of PDAmb (potato dextrose agar [Difco Laboratories, Detroit] supplemented with $0.1 \mathrm{~g} / \mathrm{liter}$ of L-methionine [E. Merck AG, Darmstadt, Germany] and $0.001 \mathrm{~g} /$ liter of biotin [Fluka AG, Buchs, Switzerland]) at $4{ }^{\circ} \mathrm{C}$ in the dark.

Two diagnostic criteria were used in this study to test for virus infection of $C$. parasitica: the white culture type and the transmissibility of the white culture type to an orange strain. The white
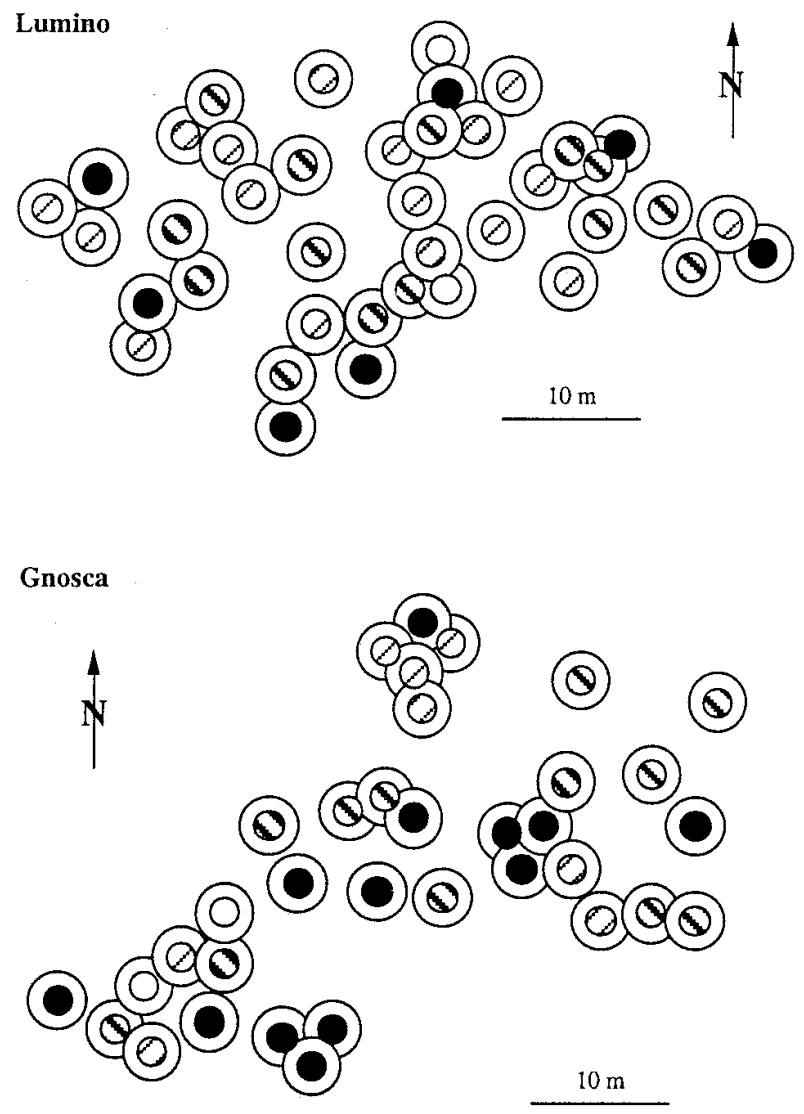

Fig. 2. Spatial distribution of the chestnut stumps in Lumino and Gnosca. The blight incidence in 1994 is indicated by the portion of 10-year-old Castanea sativa sprouts $(N=850)$ that harbored at least one Cryphonectria parasitica canker. Open circles represent stumps with $\leq 25 \%$ infected sprouts; hatched circles are stumps with $>25 \%$ to $\leq 50 \%$ (light hatching) or with $>50 \%$ to $\leq 75 \%$ (dark hatching) infected sprouts; solid circles represent stumps with $>75 \%$ infected sprouts. 
cultural appearance of $C$. parasitica is correlated with the infection of the fungus by the European hypovirus $(18,20,44)$. To assess culture characteristics, all $C$. parasitica isolates were grown on PDA plates at $25^{\circ} \mathrm{C}$ in the dark for 7 days, followed by incubation under daylight on the laboratory bench for 5 days. Under these conditions, virus-free isolates developed a clearly discernible orange pigmentation, whereas virus-infected isolates remained white. Each white isolate was tested for its ability to convert compatible orange strains in the course of the vegetative compatibility tests.

Vegetative compatibility. Tests for vegetative compatibility were performed essentially as described by Anagnostakis (4), except that conidia instead of mycelia were used for inoculations. Strains were paired about $3 \mathrm{~mm}$ apart on PDA plates and incubated as described above. Vegetative compatibility was assessed according to the merging/barrage response (4). Merging of the two colonies indicated compatible strains, whereas the formation of a barrage line between the colonies indicated incompatible strains. All isolates were paired with vegetative compatibility tester strains belonging to the Swiss VCGs 1 to 5 (13). New vegetative compatibility tester strains were selected from isolates that were incompatible with the former tester strains. Only strains showing orange culture morphology were used as vegetative compatibility testers. If white strains showed unclear reactions, single conidial isolates exhibiting orange culture morphology were used (1). Vegetative compatibility tests were repeated at least twice for each isolate. Preliminary results of VCG determination had been reported earlier (15) and were reevaluated in this study using the above procedures.

The diversity of VCGs was determined by the ratio of the number of VCGs to the sample size $(S / N)$, and by the Shannon diversity index $\left(\mathrm{H}^{\prime}\right)$ as described by Anagnostakis et al. (8). $\mathrm{H}^{\prime}$ was calculated as $-\Sigma p_{i} \ln p_{i}$, in which $p_{i}$ is the frequency of the $i$ th VCG.

Hypovirulence conversion tests. Orange and white strains of the six most common VCGs were used as recipient or donor strains of the hypovirus to determine conversion rates between the groups. Ten orange and 10 white strains were randomly selected

TABLE 1. Number of orange and white isolates of Cryphonectria parasitica among vegetative compatibility groups (VCGs) in Lumino and Gnosca in 1990

\begin{tabular}{|c|c|c|c|c|c|c|}
\hline \multirow[b]{2}{*}{$\mathrm{VCG}^{\mathrm{a}}$} & \multicolumn{3}{|c|}{ Lumino } & \multicolumn{3}{|c|}{ Gnosca } \\
\hline & Orange & White & Total & Orange & White & Total \\
\hline 1 & 9 & 10 & 19 & 12 & 8 & 20 \\
\hline 2 & 6 & 5 & 11 & 6 & 2 & 8 \\
\hline 3 & 10 & 21 & 31 & 8 & 3 & 11 \\
\hline 4 & 1 & 3 & 4 & 0 & 0 & 0 \\
\hline 6 & 0 & 0 & 0 & 2 & 3 & 5 \\
\hline 7 & 1 & 4 & 5 & 1 & 1 & 2 \\
\hline 8 & 0 & 0 & 0 & 2 & 0 & 2 \\
\hline 9 & 2 & 0 & 2 & 0 & 1 & 1 \\
\hline 10 & 1 & 0 & 1 & 0 & 1 & 1 \\
\hline 11 & 1 & 0 & 1 & 1 & 1 & 2 \\
\hline 16 & 0 & 1 & 1 & 0 & 1 & 1 \\
\hline 17 & 0 & 2 & 2 & 0 & 0 & 0 \\
\hline 20 & 2 & 4 & 6 & 2 & 2 & 4 \\
\hline Single VCGs ${ }^{\mathrm{b}}$ & 2 & 1 & 3 & 3 & 2 & 5 \\
\hline \multirow[t]{2}{*}{ All isolates } & 35 & 51 & 86 & 37 & 25 & 62 \\
\hline & $41 \%$ & $59 \%$ & $100 \%$ & $60 \%$ & $40 \%$ & $100 \%$ \\
\hline$S^{\mathrm{c}}$ & 11 & 9 & 14 & 11 & 12 & 16 \\
\hline$S / N$ & 0.31 & 0.18 & 0.16 & 0.30 & 0.48 & 0.26 \\
\hline $\mathrm{H}^{\prime d}$ & 1.95 & 1.76 & 1.94 & 1.95 & 2.18 & 2.18 \\
\hline
\end{tabular}

a Swiss system of vegetative compatibility grouping; VCGs 1 to 4 correspond to the groups determined by Bazzigher et al. (13).

b VCGs with only one isolate.

c Number of VCGs.

d Shannon diversity index, $\mathrm{H}^{\prime}=-\Sigma p_{i} \ln p_{i}$ in which $p_{i}$ is the frequency of the $i$ th VCG. from each VCG. Some isolates were selected more than once, if 10 strains were not available for a particular VCG and culture type. The selected strains were used pairwise, giving 10 conversion tests for each of the 36 possible VCG combinations. Pairings were made by inoculating white and orange strains $5 \mathrm{~mm}$ apart at the edge of a PDA plate (9 $\mathrm{cm}$ in diameter); cultures were incubated at $25^{\circ} \mathrm{C}$ in the dark for 14 days. Conversion was considered successful if the orange strain developed the white culture characteristics (7). Conversion tests were performed twice for each pair of isolates. Conversion rates were calculated as percentage of successful conversions based on the total number of pairings. Cluster analysis was performed based on the conversion matrix using DATA DESK version 4.2 (Data Description Inc., Ithaca, NY).

Mating type tests. All isolates were crossed with each of the two mating type testers, M1115 and M1297, of C. parasitica (43) on autoclaved pieces of Castanea sativa stems according to Anagnostakis (4). Orange isolates were inoculated together with the mating tester (one strain on each site of the stem) and incubated at $25^{\circ} \mathrm{C}$ under a 16 -h photoperiod $(2,500 \mathrm{~lx})$ for 14 days. Sterile water was added to the plates, and the conidia produced by both strains were suspended and distributed over the stem segment to incite mating. To test white strains, which are usually female sterile (4), the mating plates were inoculated first with the tester strain alone and incubated as above. After 2 weeks, a suspension of conidia of the white strain was added and distributed over the stem segment to fertilize the tester strain. Conidia of the white strains were produced by incubating the strains on PDA plates at $25^{\circ} \mathrm{C}$ under high light $(10,000 \mathrm{~lx})$ for 14 days (32). Mating plates were incubated at $18^{\circ} \mathrm{C}$ under a 8 -h photoperiod $(2,500 \mathrm{~lx})$ and were examined for the occurrence of perithecia after 2 and 4 months. Each cross was performed twice. Because self-fertilization has been observed in $C$. parasitica $(4,39)$, both mating type tester strains were tested in each set of crosses for their ability to produce perithecia alone. Selfing was not observed with the two mating type testers.

Spatial distribution of hypovirulence and VCGs. Orange and white strains, as well as the VCGs, were tested for their aggregation in the two plots Lumino and Gnosca. Spatial pattern analysis was performed as described by Milgroom et al. (40). Two matrices were built. The first matrix, D, contained the distances between all sprout clusters on which each individual canker was found. Elements of D were called $d_{\mathrm{ij}}$, which represents the distance between the cankers $i$ and $j$. The second matrix, $G$, indicated whether individuals belonged to the same culture type or VCG. Elements in matrix $\mathrm{G}$ were called $g_{\mathrm{ij}}$ and scaled in two different ways. The first scale was designed so each culture type or VCG that occurred more than once contributed equally to the analysis. In this case, the elements $g_{\mathrm{ij}}$ of $\mathrm{G}$ were defined as $1 / n_{k}\left(n_{k}-1\right)$ and 0 . The equation $1 / n_{k}\left(n_{k}-1\right)$ is when $\mathrm{i} \neq \mathrm{j}$, and individual cankers $\mathrm{i}$ and $\mathrm{j}$ have the same characteristics, $k$ (i.e., culture type or VCG). $n_{k}$ is the number of cankers with characteristics $k$ found in the population. Zero is when $\mathrm{i} \neq \mathrm{j}$, and individual cankers $\mathrm{i}$ and $\mathrm{j}$ have

TABLE 2. Hypovirulence conversion rates (\%) among six different vegetative compatibility groups (VCGs) of Cryphonectria parasitica obtained by pairing white (donor) with orange (recipient) strains

\begin{tabular}{lrrrrrr}
\hline \multirow{2}{*}{$\begin{array}{l}\text { Donor strain } \\
\text { VCG }\end{array}$} & \multicolumn{7}{c}{ Recipient strain VCG } \\
\cline { 2 - 6 } 1 & 2 & 3 & 6 & 7 & 20 \\
\hline 2 & $100^{\mathrm{a}}$ & 10 & 35 & 10 & 40 & 100 \\
3 & 15 & 100 & 100 & 10 & 10 & 55 \\
6 & 35 & 100 & 100 & 0 & 5 & 40 \\
7 & 0 & 0 & 0 & $100^{\mathrm{b}}$ & $8^{\mathrm{b}}$ & 5 \\
20 & 100 & 25 & 25 & 35 & 100 & 100 \\
\hline a Based on 20 pairings unless indicated. & & & \\
b Twelve pairings, because not enough strains were available for these com- \\
binations.
\end{tabular}


different characteristics, $k$ (i.e., culture type or VCG). The second scale was designed so each individual contributed equally to the analysis, in which case $g_{\mathrm{ij}}$ was 1 for cankers $\mathrm{i}$ and $\mathrm{j}$ with the same characteristics, and 0 otherwise. The test statistic was calculated as the sum of the cross-products, $\Gamma$, of the elements D and G. This observed result was compared with the null distribution of $\Gamma(p)$, derived by randomly assigning culture type or vegetative compatibility type to isolates 1,000 times.

Disease development over 4 years. In 1990, all living sprouts were numbered, and the center of every sprout cluster was mapped. Before the study was started, all dead sprouts were removed. Between 1990 and 1994, the two plots were examined regularly in August. Growth of the sprouts was followed by measuring the diameter at breast height (dbh) in 1990, 1992, and 1994. Yearly, all sprouts were checked for cankers and survival. $C$. parasitica was noted as the cause of mortality, if it caused the death of the distal parts of the sprout. Death of the sprout without apparent contribution of $C$. parasitica was attributed to competition. All cankers on living sprouts located up to $2 \mathrm{~m}$ above ground level were noted. Each canker was measured by determining the extension of the altered bark morphology (bark swollen, sunken, split, and sloughed, alone or in combination) or discoloration of the bark in direction of the stem axis. Encircling cankers were noted. When sprouts died during the study, the cankers were no longer measured. During the study, dead sprouts were not removed from the plots.

\section{RESULTS}

Population structure of $\boldsymbol{C}$. parasitica in 1990. In the beginning, 190 randomly selected cankers (100 from Lumino and 90 from Gnosca) in the two study plots were sampled. A total of 148 cankers yielded $C$. parasitica isolates, whereas the other 42 cankers yielded other fungi or no fungi. Fifty-nine and $40 \%$ of the $C$. parasitica isolates from Lumino and Gnosca, respectively, had the white culture type (Table 1).

The VCG of each C. parasitica isolate from 1990 was determined. A total of 14 VCGs were identified among the 86 isolates in Lumino compared with 16 VCGs among the 62 isolates in Gnosca (Table 1). In combination, 21 different VCGs were found in the two plots. The three VCGs 1,2 , and 3 were codominant in both plots with 71 and $63 \%$ of the total isolates in Lumino and Gnosca, respectively. Many VCGs were represented by only one or two isolates. Five orange and three white isolates were vegetatively incompatible with all other testers, thus representing eight

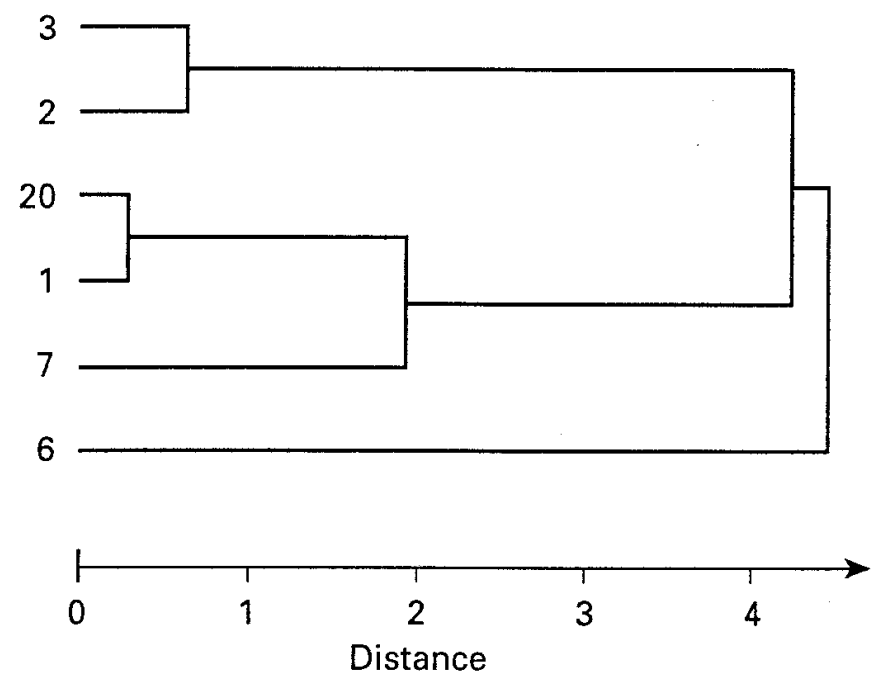

Fig. 3. Dendrogram of the six most common vegetative compatibility groups (VCGs) of Cryphonectria parasitica. Complete linkage clustering was generated based on the hypovirulence conversion rates between the VCGs.
VCGs with only one isolate. Ten VCGs, including the most frequent ones, contained both orange and white isolates. Six VCGs were represented only by orange strains and five VCGs only by white strains. Orange strains typically gave clear and consistent vegetative compatibility reactions, i.e., the unknown isolate was clearly compatible with one vegetative compatibility tester strain and incompatible with all others. Vegetative compatibility tests involving white strains were more difficult to assess. Several strains required the isolation of a single conidial isolate with orange culture morphology before accurate vegetative compatibility tests could be performed. All white strains belonging to a VCG without orange strains were tested in this manner. Each white strain converted the tester of the same VCG to the white phenotype. Pairings between strains of VCG 2 and 3 and VCG 1 and 20 reproducibly resulted in weak vegetative compatibility reactions, as indicated by a faint barrage zone.

The $S / N$ ratios and Shannon diversity indices $\left(\mathrm{H}^{\prime}\right)$ were calculated for both populations to estimate the diversity of VCGs within the two $C$. parasitica populations. In Lumino, both $S / N$ and $\mathrm{H}^{\prime}$ were higher for orange isolates than for white isolates, whereas, in Gnosca, $S / N$ and $\mathrm{H}^{\prime}$ were lower for orange than for white isolates (Table 1). If orange and white isolates were scored together, the diversity of the VCGs was higher in Gnosca $\left(S / N=0.26 ; \mathrm{H}^{\prime}=\right.$ 2.18) than in Lumino $\left(S / N=0.16 ; \mathrm{H}^{\prime}=1.94\right)$.

The ability of white strains to convert orange strains was tested for the six most frequent VCGs. The obtained hypovirulence conversion rates are shown in Table 2. If orange and white strains belonged to the same VCG, conversions were always $100 \%$ successful. Some pairings between white and orange strains of different VCGs yielded $100 \%$ conversion as well, independent of which VCG provided donor or recipient strains (as evident from pairings between VCGs 2 and 3 and VCGs 1 and 20). Conversion rates between VCG 7 and VCG 1 or 20 was $100 \%$ if the donor strains belonged to VCG 7 , but only $40 \%$ if VCG 7 represented the recipient strains. Conversion rates of all other combinations ranged from 0 to $55 \%$ and showed similar rates in reciprocal pairings. Very low or no conversion rates were observed in pairings involving strains of VCG 6 . In most combinations with conversion rates below $100 \%$, only one of the two identical pairings resulted in conversion of the orange strain. This was the case in an average of $87 \%$ of all these pairings. Complete linkage clustering was performed to estimate relatedness between the six VCGs, based on the conversion rates of the recipient strains (Fig. 3). According to this analysis, VCGs 2 and 3, as well as 1, 7, and 20, are clustered together, whereas VCG 6 is separated. Final clustering was the same if the data of the donor strains were used (data not shown).

The distribution of the two mating types of $C$. parasitica among white and orange isolates and among VCGs of the two populations in 1990 is summarized in Tables 3 and 4. The mating type of practically all orange isolates and more than $90 \%$ of all white isolates was identified. Eleven isolates in Lumino and 14 in Gnosca produced perithecia with both mating type testers. In Gnosca, the two mating types were found in almost 1:1 ratio. In contrast, one mating type (MAT1-1) was dominant in Lumino, resulting in a ratio that was significantly different than $1: 1(P \leq 0.05)$. The dominance was due to a much higher portion of white MAT1-1 than white MAT1-2 isolates. In Lumino, VCG 7 and 20 exclu-

TABLE 3. Mating type of orange and white isolates of Cryphonectria parasitica in the populations Lumino and Gnosca in 1990

\begin{tabular}{lrrrrrrrr}
\hline & \multicolumn{3}{c}{ Lumino } & & \multicolumn{3}{c}{ Gnosca } \\
\cline { 2 - 4 } \cline { 7 - 8 } \cline { 6 - 8 } & Orange & White & Total & & Orange & White & Total \\
\hline MAT1-1 & 15 & 29 & 44 & & 14 & 7 & 21 \\
MAT1-2 & 12 & 15 & 27 & & 12 & 12 & 24 \\
MAT1-1 and MAT1-2 & 8 & 3 & 11 & & 10 & 4 & 14 \\
No perithecia formed & 0 & 4 & 4 & & 2 & 3 \\
\hline
\end{tabular}

a Perithecia were formed with both mating type testers. 
sively yielded MAT1-1 isolates (Table 4). Both mating types were detected in the most common VCGs, 1, 2, and 3.

Culture types (orange or white) and VCGs of the isolates were tested for nonrandom distribution in each plot using spatial pattern analysis (Table 5). Two different weighting options were used: each characteristic (culture type, VCG) contributed equally to the statistic or each $C$. parasitica isolate contributed equally to the statistic. For both weighting options, the orange and white strains were found to be nonrandomly distributed $(P<0.05)$ in Gnosca, but not in Lumino. VCGs showed nonrandom distribution in Gnosca, independent of the weighting options. In Lumino, however, a significant nonrandom distribution $(P<0.05)$ was found for VCGs when each VCG contributed equally to the statistic. The distribution in Lumino did not deviate significantly from randomness if each isolate was weighted equally (Table 5).

Canker growth and dynamics of $\boldsymbol{C}$. parasitica over 4 years. During the observation period, cankers that yielded orange isolates in the beginning of the study killed 34 and $30 \%$ of their hosts in Lumino and Gnosca, respectively. The corresponding host mortality for cankers yielding white strains was 14 and $8 \%$ for the two plots. The growth rate of cankers on living sprouts decreased gradually in both plots from 1990 to 1994 independently, whether they yielded an orange or white strain in 1990 (Fig. 4). Cankers harboring white $C$. parasitica isolates expanded slower than cankers harboring orange isolates (Fig. 4). Between 1990 and 1993, the cankers in Lumino that yielded white culture types in 1990 grew significantly slower $(P \leq 0.05)$ than the cankers yielding orange culture types. In Gnosca, however, only between 1992 and 1993, cankers yielding white culture types grew significantly slower $(P \leq 0.05)$ than cankers yielding orange culture types.

To see whether the decrease in canker growth was represented by an increase of white isolates in the $C$. parasitica population, all cankers that yielded $C$. parasitica in 1990 were sampled again in 1992 and 1994 (Table 6). Sixteen cankers in Lumino and 18 in Gnosca repeatedly yielded only orange isolates. From all other cankers, white culture types were isolated at least once during the 4 years of investigation. In 1992, 73\% (Lumino) and 76\% (Gnosca) of the cankers yielded the same culture type as in 1990. When the isolates of 1992 and 1994 were compared, 63\% (Lumino and Gnosca) of the cankers yielded isolates with the same culture type in both years (Table 6). The ratio of orange to white isolates was always higher in Gnosca than in Lumino.

Fast and nongrowing cankers. In 1992, C. parasitica was isolated from the 30 cankers in each plot that showed the fastest (mean growth $>30 \mathrm{~cm}$ in 2 years) and slowest (no growth in 2 years) growth rates between 1990 and 1992 (Table 7). More than

TABLE 4. Mating type ratio (MAT1-1:MAT1-2) of Cryphonectria parasitica isolates belonging to different vegetative compatibility groups (VCGs) in the populations Lumino and Gnosca

\begin{tabular}{lccccc}
\hline & \multicolumn{2}{c}{ Lumino } & & \multicolumn{2}{c}{ Gnosca } \\
\cline { 6 - 6 } \cline { 5 - 6 } VCG & $\begin{array}{c}\text { No. } \\
\text { identified }^{\mathrm{a}}\end{array}$ & $\begin{array}{c}\text { Mating type } \\
\text { ratio }\end{array}$ & & $\begin{array}{c}\text { No. } \\
\text { identified }\end{array}$ & $\begin{array}{c}\text { Mating type } \\
\text { ratio }\end{array}$ \\
\hline 1 & 16 & $9: 7$ & & 13 & $5: 8$ \\
2 & 7 & $4: 3$ & & 4 & $2: 2$ \\
3 & 26 & $15: 11$ & & 10 & $5: 5$ \\
6 & $\mathrm{NF}^{\mathrm{b}}$ & & & 4 & $0: 4$ \\
7 & 5 & $5: 0$ & & 2 & $1: 1$ \\
20 & 4 & $4: 0$ & & 1 & $1: 0$ \\
Others $^{\mathrm{c}}$ & 13 & $7: 6$ & & 11 & $7: 4$ \\
& & & & & \\
Total & 71 & $44: 27^{\mathrm{d}}$ & 45 & $21: 24^{\mathrm{e}}$ \\
\hline
\end{tabular}

${ }^{a}$ Isolates sexually compatible with both testers not included.

${ }^{\mathrm{b}}$ VCG not found in this population.

${ }^{c}$ Others included eight VCGs for Lumino and nine for Gnosca.

${ }^{\mathrm{d}}$ Observed mating type ratio is significantly different from 1:1 $(P<0.05$; chi-square test).

e Mating type ratio is not significantly different from 1:1.
$92 \%$ of the fast growing but only $58 \%$ of the nongrowing cankers yielded $C$. parasitica isolates. Both fast and nongrowing cankers yielded orange and white $C$. parasitica culture types. However, from nongrowing cankers, often no $C$. parasitica could be isolated. In Lumino and Gnosca, fast growing cankers yielded a higher portion of orange isolates than nongrowing cankers. White strains were isolated in similar amounts from both canker types. In both plots, a significant number of fast growing cankers harbored white C. parasitica isolates. Between 1992 and 1994, the fast growing cankers showed a reduced growth rate compared with the 2 previous years. This reduction was observed independently of whether they harbored orange or white culture types. Until the end of the study in 1994, none of the cankers described as nongrowing resumed growth, even if they were found to be harboring orange $C$. parasitica isolates.

Disease development over 4 years. A total of 850 Castanea sativa sprouts growing from 76 stumps were examined between 1990 and 1994. The number of chestnut sprouts per stump varied between 2 and 28. Between 1990 and 1994, the portion of cankered sprouts increased from 37 to $58 \%$ at Lumino and from 37 to $61 \%$ at Gnosca (Table 8). Within the observation period, the number of cankers almost doubled, resulting in many sprouts with several cankers. The chestnut blight incidence among the different stumps varied greatly (Fig. 2). The portion of encircling cankers increased only slightly during the 4-year investigation period. Many of them were not lethal. In both plots, chestnut blight killed $15 \%$ of the Castanea sativa sprouts. An additional 23\% (Lumino) and 19\% (Gnosca) of the sprouts died for other reasons, possibly due to competition (Table 8). Between 1990 and 1994, the mean dbh of all living chestnuts increased by $2.6 \mathrm{~cm}$ in both Lumino and Gnosca (Fig. 5). At the end of the investigation, the dbh of the trees ranged from 2 to 16 $\mathrm{cm}$ in Lumino and from 2 to $14 \mathrm{~cm}$ in Gnosca. The sprouts that were killed by chestnut blight or competition had a significantly lower dbh in 1990 than the sprouts that survived between 1990 and 1994.

The growth rates of all cankers were measured in both plots over 4 years by determining their expansion in the direction of the main stem axis. In the first year of investigation, the mean growth rate of all cankers on living sprouts was $6.0 \mathrm{~cm}$ in Lumino and 7.6 $\mathrm{cm}$ in Gnosca. Although cankers on dead sprouts were eliminated from the data sets, the average growth rate of the cankers dropped to $0.7 \mathrm{~cm}$ in Lumino and to $1.1 \mathrm{~cm}$ in Gnosca within the 4 years of

TABLE 5. Spatial pattern analysis for culture types and vegetative compatibility groups (VCGs) of Cryphonectria parasitica in Lumino and Gnosca in 1990

\begin{tabular}{llllll}
\hline Plot & $N^{\mathrm{a}}$ & $V_{2}^{\mathrm{b}}$ & $\Gamma / V_{2}^{\mathrm{c}}$ & $\Gamma(p)^{\mathrm{d}}$ & $P^{\mathrm{e}}$ \\
\hline $\begin{array}{l}\text { Culture types } \\
\text { Lumino }^{\mathrm{f}}\end{array}$ & 86 & 2 & 15.79 & 16.04 & 0.102 \\
Gnosca $^{\mathrm{f}}$ & 62 & 2 & 15.50 & 16.62 & 0.012 \\
Lumino $^{\mathrm{g}}$ & 86 & 2 & 15.97 & 16.02 & 0.312 \\
Gnosca $^{\mathrm{g}}$ & 62 & 2 & 15.79 & 16.58 & 0.007 \\
& & & & & \\
VCGs $_{\text {Cumino }}^{\mathrm{h}}$ & 80 & 8 & 12.18 & 15.92 & 0.004 \\
Gnosca $^{\mathrm{h}}$ & 54 & 8 & 16.07 & 16.51 & 0.422 \\
Lumino $^{\mathrm{g}}$ & 80 & 8 & 15.29 & 16.00 & 0.080 \\
Gnosca $^{\mathrm{g}}$ & 54 & 8 & 16.55 & 16.58 & 0.468 \\
\hline
\end{tabular}

${ }^{a}$ Number of isolates that were included in the analysis.

${ }^{b}$ Number of culture types or VCGs that occurred more than once in each plot.

c Observed mean distance (in meters) of isolates with the same culture type or VCG.

${ }^{\mathrm{d}}$ Expected mean distance under the assumption that culture types or VCGs are assigned at random.

e Probability of the observed mean distance compared with the mean distance $(\Gamma(p))$ that was expected after 1,000 randomizations under the null hypothesis of no aggregation.

${ }^{\mathrm{f}}$ Both culture types weighted equally.

$\mathrm{g}$ Each isolate weighted equally.

h Each VCG weighted equally. 
investigation. The portion of cankers with a high growth rate $(\geq 10$ $\mathrm{cm}$ per year) constantly decreased, whereas the portion of cankers with a low growth rate ( $\leq 2 \mathrm{~cm}$ per year) increased considerably (data not shown). A total of $12.8 \%$ of all cankers on living sprouts did not extend at all between 1990 and 1994.

A total of 398 new cankers developed in the two plots during the investigation. The diameter of newly infested sprouts did not deviate significantly from the diameter of all living sprouts. Newly developing cankers always exhibited high growth rates of more than $10 \mathrm{~cm} /$ year in their first year (Table 9). But in their second year, the cankers reduced their growth rates significantly to less than 4 $\mathrm{cm}$. In the fourth year, their average growth rate was $1.5 \mathrm{~cm}$.

\section{DISCUSSION}

Population structure of $\boldsymbol{C}$. parasitica. A total of 21 VCGs were identified at the two study sites. Fourteen VCGs were found in Lumino and 16 in Gnosca. In the two populations, the same

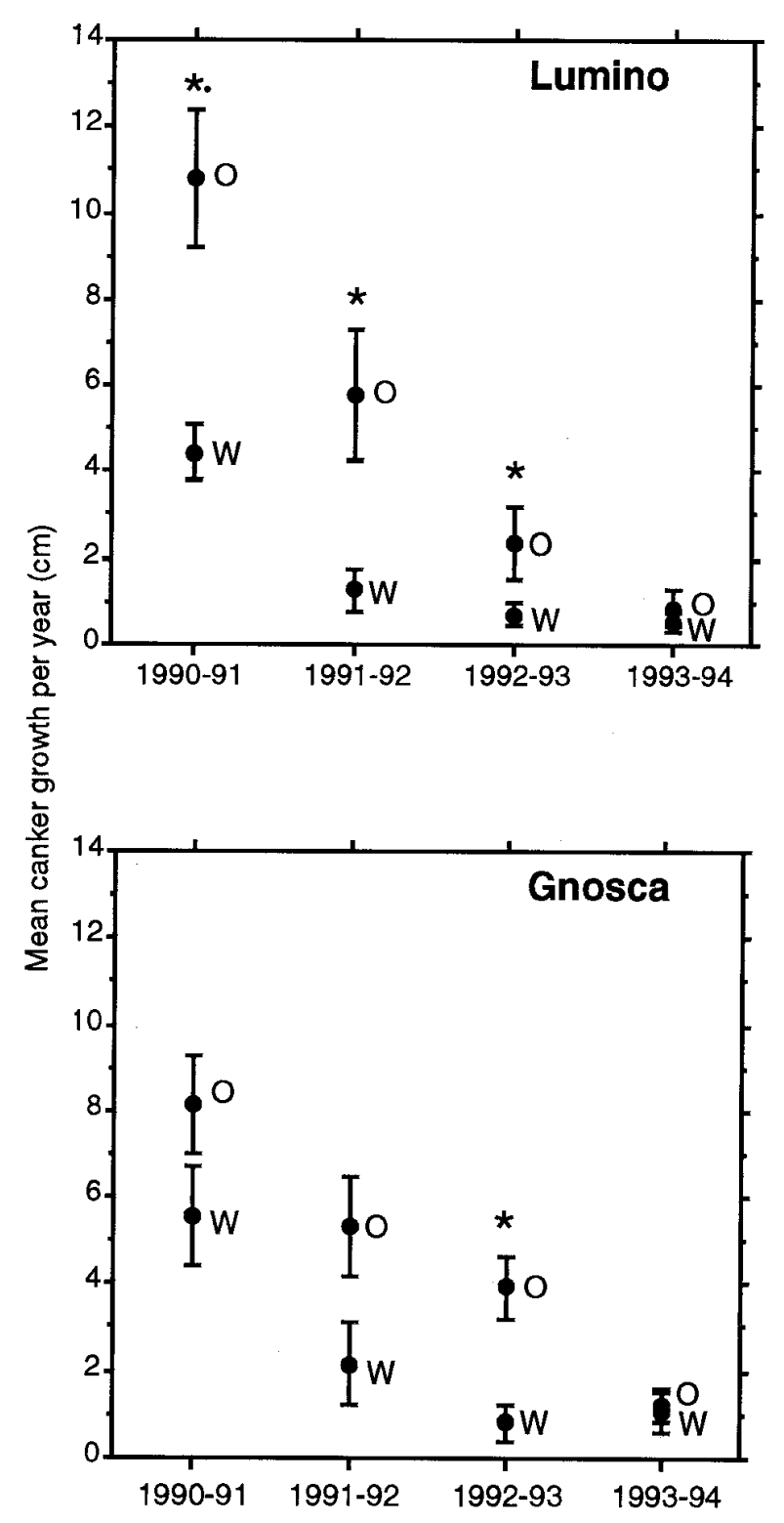

Fig. 4. Growth of chestnut blight cankers on Castanea sativa. Mean canker growth rates ( \pm standard error) in Lumino (86 cankers) and Gnosca (62 cankers) between 1990 and 1994 of randomly selected cankers that yielded orange (O) or white (W) Cryphonectria parasitica isolates in 1990 (asterisk, $P \leq 0.05$, Student's two-tailed $t$ test). Cankers on dead sprouts are excluded from the analysis. three VCGs were dominant with 63 and $71 \%$ of the isolates, respectively. The same three VCGs already dominated in a survey conducted between 1976 and 1979 in the Ticino (13). At that time, 10 VCGs among 72 isolates were identified (six VCGs that occurred more than once; D. Rigling, unpublished data). Although different sampling methods were used, our study indicates that VCG diversity has increased in this region. The increase of VCG diversity might be because of (i) sexual recombination between resident VCGs; (ii) introduction of new VCGs, probably from nearby Italy; or (iii) mutations that generated new VCGs.

The diversity of VCGs calculated as $S / N$ ratio and the Shannon diversity index $\left(\mathrm{H}^{\prime}\right)$ was higher in the Gnosca than in the Lumino population. The number of VCGs and the $\mathrm{H}^{\prime}$ indices suggest that VCG diversity is lower in Swiss than in most United States populations. In similar small area studies in Virginia (34), West Virginia (40), and Connecticut (9), up to 21, 27, and 48 different VCGs were reported, respectively. The VCG diversity index of two small area populations in Virginia and Connecticut yielded an $\mathrm{H}^{\prime}$ of 2.76 and 2.8 (9), which is higher than found in this study with an $\mathrm{H}^{\prime}$ of 1.94 and 2.18. An accurate comparison, however, is difficult because of different sample sizes and sampling methodologies used in each study. Our results reveal a greater diversity in some European $C$. parasitica populations than formerly estimated (30).

TABLE 6. The same naturally occurring Cryphonectria parasitica cankers in Lumino and Gnosca were sampled in 1990, 1992, and 1994a

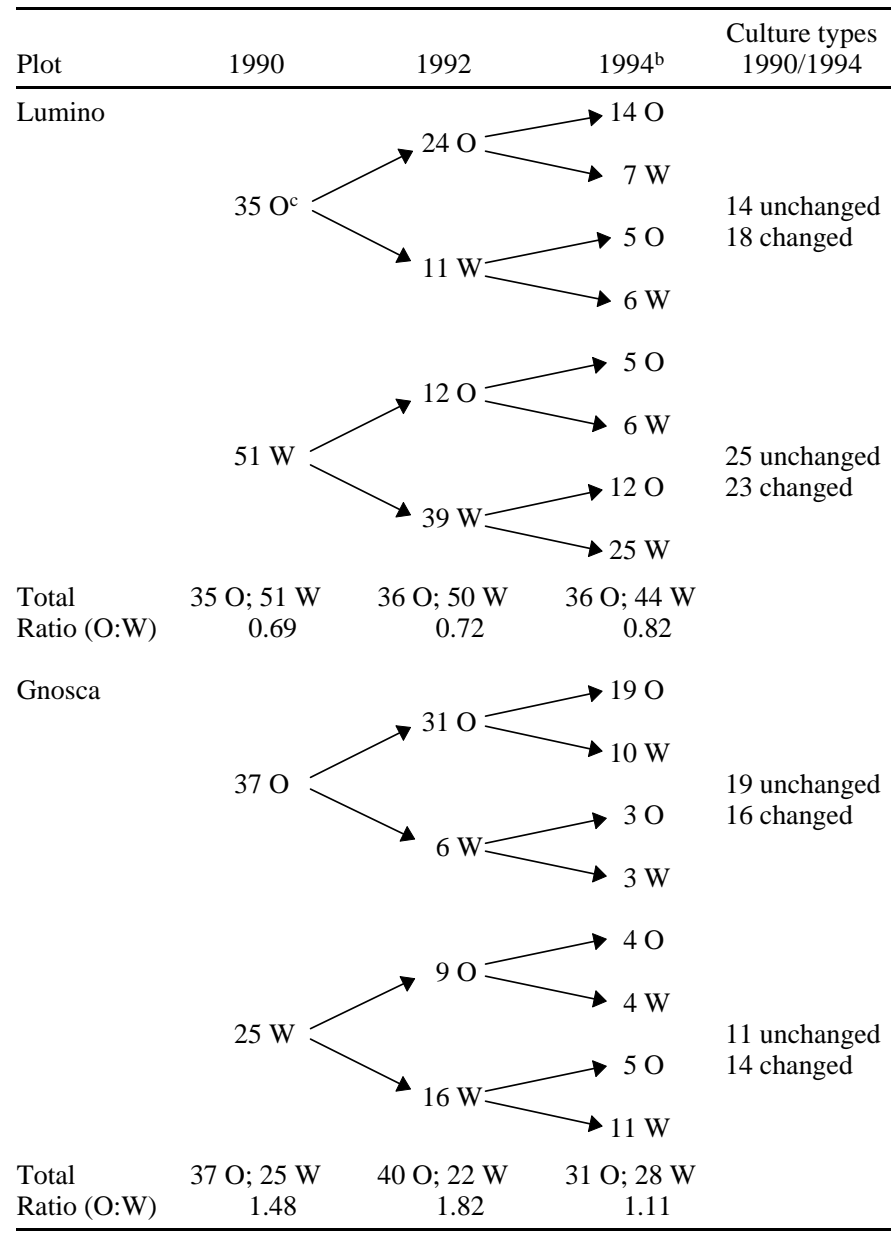

a Several cankers yielded different culture types in the 3 years. Numbers represent cankers on Castanea sativa that yielded orange (O) or white (W) culture types.

${ }^{b}$ Six cankers in Lumino and three in Gnosca yielded no Cryphonectria parasitica in 1994.

c e.g., Out of 35 cankers that yielded orange $(\mathrm{O})$ culture types in Lumino in 1990, 24 yielded the same culture type (O) in 1992, whereas 11 yielded a white culture type (W). 
White strains were detected at variable frequencies in all major VCGs. In addition, several VCGs were represented only by white isolates. This finding is surprising because the hypovirus, responsible for the white phenotype, is not transmitted into the sexual ascospores, which are thought to be the main source for new VCGs (4). If VCG diversity is limiting the spread of the hypovirus, white strains are not expected to be frequent in rare VCGs. This study suggests that the hypovirus is very effectively transmitted into rare VCGs, or that mutations at the vic loci in virusinfected strains lead to the establishment of white isolates in rare VCGs. Instability of VCGs was observed in $C$. parasitica after ultraviolet treatment (45). However, it is not known if this phenomenon occurs under natural conditions and in virus-infected strains.

Hypovirulence conversion studies between the six most common VCGs (comprising more than $80 \%$ of the isolates) also suggest that vegetative incompatibility is probably not a major barrier for the spread of the hypovirus in southern Switzerland. Five of these six VCGs fall into two clusters, with conversion rates of almost $100 \%$ within each cluster. Clustering of VCGs into conversion groups was observed in United States $(33,34)$ and Italian populations (23). Conversion group diversity is probably a more relevant parameter than VCG diversity to assess the potential for spread of the hypovirus in C. parasitica populations.

In addition to VCG, we also included mating type as a phenotypic marker to analyze the populations. The two mating types were found in both plots, suggesting that sexual reproduction is occurring in these populations. In fact, perithecia, the sexual fruiting bodies of $C$. parasitica, have been observed, but they were not very frequent (14). Several isolates were sexually compatible with both mating types, and many were also able to pro- duce perithecia alone (14). This phenomenon may be the result of heterokaryosis (2), self-fertilization, or mixed cultures. Self-fertilization was found in United States (39), as well as Swiss (16), C. parasitica populations and could contribute, together with asexual reproduction, to a clonal population structure. Current investigations suggest that self-fertilized perithecia of $C$. parasitica produce both mating types in a 1:1 ratio and, thus, do not affect the mating type ratio in a population (D. Rigling, M. Bissegger, and U. Heiniger, unpublished data).

The hypovirus infection rate of the fungal population in Lumino (59\%) was considerably higher than in Gnosca (40\%). The differences between the population structures might account for this observation. In Gnosca, the VCG diversity was higher compared with Lumino. The random distribution of the VCGs in Gnosca might be a further obstacle for the spread of the hypovirus in this population. In Lumino, on the other hand, aggregation of VCGs and a high portion of white strains were detected. The aggregation of VCGs and the unbalanced mating type ratio in Lumino indicate that a portion of this population reproduced clonally. This would favor the movement of the hypovirus within the fungal host population (38). Interestingly, white strains were significantly $(P$ $<0.01$ ) aggregated in Gnosca, but not in Lumino. One possible explanation for this is that, in Gnosca, white strains were introduced only in a few spots from where the hypovirus slowly spread. In contrast, multiple introductions of white strains from the surrounding chestnut forests could result in a more random distribution, as observed in Lumino.

The distribution of VCGs and mating types among orange and white isolates was very similar in both populations. This indicates a rather homogeneous infection of the two $C$. parasitica populations by the virus that causes the white phenotype and hypoviru-

TABLE 7. Culture types of Cryphonectria parasitica isolates and growth rates of fast and nongrowing cankers in Lumino and Gnosca on Castanea sativa

\begin{tabular}{|c|c|c|c|c|c|c|c|}
\hline \multirow[b]{3}{*}{ Canker type ${ }^{a}$} & \multirow[b]{3}{*}{ Culture type in $1992^{b}$} & \multicolumn{3}{|c|}{ Lumino } & \multicolumn{3}{|c|}{ Gnosca } \\
\hline & & \multirow{2}{*}{$\begin{array}{c}\text { No. of } C \text {. parasitica } \\
\text { isolates }\end{array}$} & \multicolumn{2}{|c|}{ Mean growth rate $(\mathrm{cm})$} & \multirow{2}{*}{$\begin{array}{c}\text { No. of } C \text {. parasitica } \\
\text { isolates }\end{array}$} & \multicolumn{2}{|c|}{ Mean growth rate $(\mathrm{cm})$} \\
\hline & & & 1990-1992 & 1992-1994 & & 1990-1992 & 1992-1994 \\
\hline \multirow{4}{*}{$\begin{array}{l}\text { Fast growing } \\
\quad(\varnothing 90-92=34.5 \mathrm{~cm})\end{array}$} & Total & $30(100 \%)$ & $33.0 \pm 2.9$ & $7.2 \pm 2.4$ & $30(100 \%)$ & $36.0 \pm 3.3$ & $8.9 \pm 7.0$ \\
\hline & Orange & $12(40 \%)$ & $34.5 \pm 5.6$ & $5.1 \pm 4.3$ & $14(46 \%)$ & $33.9 \pm 4.4$ & $15.8 \pm 15.4$ \\
\hline & White & $18(60 \%)$ & $32.0 \pm 3.7$ & $8.6 \pm 2.9$ & $11(37 \%)$ & $36.6 \pm 7.5$ & $3.8 \pm 4.0$ \\
\hline & No C. parasitica & 0 & $\ldots$ & $\ldots$ & $5(17 \%)$ & $40.6 \pm 9.3$ & $0.8 \pm 1.5$ \\
\hline \multirow{4}{*}{$\begin{array}{l}\text { Nongrowing } \\
(\varnothing 90-92=0.0 \mathrm{~cm})\end{array}$} & Total & $30(100 \%)$ & 0 & $0.5 \pm 1.2$ & $30(100 \%)$ & $0.1 \pm 0.3$ & $0.1 \pm 0.3$ \\
\hline & Orange & $2(7 \%)$ & 0 & 0 & $8(27 \%)$ & 0 & $0.1 \pm 0.4$ \\
\hline & White & $15(50 \%)$ & 0 & $0.5 \pm 1.2$ & $10(33 \%)$ & $0.1 \pm 0.3$ & $0.2 \pm 0.4$ \\
\hline & No C. parasitica & $13(43 \%)$ & 0 & 0 & $12(40 \%)$ & 0 & 0 \\
\hline
\end{tabular}

a Thirty Cryphonectria parasitica cankers showing the fastest and slowest growth rates between 1990 and 1992 were examined in each plot.

${ }^{\mathrm{b}}$ Culture type of Cryphonectria parasitica isolates.

TABLE 8. Number of living, cankered, and dead chestnut (Castanea sativa) sprouts, and naturally occurring Cryphonectria parasitica cankers in Lumino and Gnosca between 1990 and 1994

\begin{tabular}{|c|c|c|c|c|c|c|c|c|}
\hline Plot & Year & $\begin{array}{l}\text { Living } \\
\text { sprouts }\end{array}$ & $\begin{array}{l}\text { Mean dbh } \mathrm{db}^{\mathrm{a}}(\mathrm{cm}) \\
\text { of living sprouts }\end{array}$ & $\begin{array}{l}\text { Sprouts with } \\
\text { cankers }^{b}\end{array}$ & Cankers $^{c}$ & $\begin{array}{c}\text { Portion of } \\
\text { encircling cankers }^{c}\end{array}$ & $\begin{array}{l}\text { Dead sprouts due to } \\
\text { C. parasitica }\end{array}$ & $\begin{array}{c}\text { Dead sprouts for } \\
\text { other reasons }\end{array}$ \\
\hline \multirow[t]{5}{*}{ Lumino } & 1990 & 410 & \multirow[t]{2}{*}{5.5} & $153(37 \%)$ & 199 & $31.2 \%$ & 0 & 0 \\
\hline & 1991 & 358 & & $181(44 \%)$ & 280 & $39.4 \%$ & 16 & 36 \\
\hline & 1992 & 324 & \multirow[t]{2}{*}{7.0} & $206(50 \%)$ & 308 & $39.4 \%$ & 31 & 55 \\
\hline & 1993 & 301 & & $227(55 \%)$ & 336 & $41.7 \%$ & 40 & 69 \\
\hline & 1994 & 256 & 8.1 & $237(58 \%)$ & 365 & $40.8 \%$ & 61 & 93 \\
\hline \multirow[t]{5}{*}{ Gnosca } & 1990 & 440 & \multirow[t]{2}{*}{4.4} & $162(37 \%)$ & 212 & $50.9 \%$ & 0 & 0 \\
\hline & 1991 & 413 & & $193(44 \%)$ & 318 & $51.6 \%$ & 15 & 12 \\
\hline & 1992 & 378 & \multirow[t]{2}{*}{5.7} & $222(50 \%)$ & 361 & $52.1 \%$ & 36 & 26 \\
\hline & 1993 & 352 & & $264(60 \%)$ & 421 & $47.0 \%$ & 48 & 40 \\
\hline & 1994 & 291 & 7.0 & $270(61 \%)$ & 444 & $47.3 \%$ & 67 & 82 \\
\hline \multirow[t]{2}{*}{ Total } & 1990 & 850 & 4.9 & $315(37 \%)$ & 411 & $41.4 \%$ & $\ldots{ }^{d}$ & $\ldots{ }^{d}$ \\
\hline & 1994 & 547 & 7.5 & $507(60 \%)$ & 809 & $44.1 \%$ & 128 & 175 \\
\hline
\end{tabular}

${ }^{a}$ Diameter at breast height.

${ }^{\mathrm{b}}$ Dead sprouts included.

${ }^{c}$ Cankers on dead sprouts included.

d All dead sprouts removed. 
lence. Our study suggests that the success of natural hypovirulence in Europe depends on the ability of the hypovirus to infect large portions of the fungal population, presumably as a result of frequent virus transmission within and between VCGs.

Disease development. The chestnut blight epidemic was followed over a period of 4 years to study the interactions between populations of Castanea sativa and $C$. parasitica. Although the two investigated $C$. parasitica populations were different in many aspects (i.e., VCG diversity, spatial distribution of VCGs, mating type ratio, and the portion of white strains), there was no considerable difference in the development of chestnut blight. In both plots, a comparable decrease in the severity of the disease was observed.

The results from our study are controversial with regard to the role of hypovirulence for the blight development in the two European chestnut forests. The increase of nongrowing cankers could be the result of hypovirulence because orange strains in active cankers were converted or because new cankers were initiated by
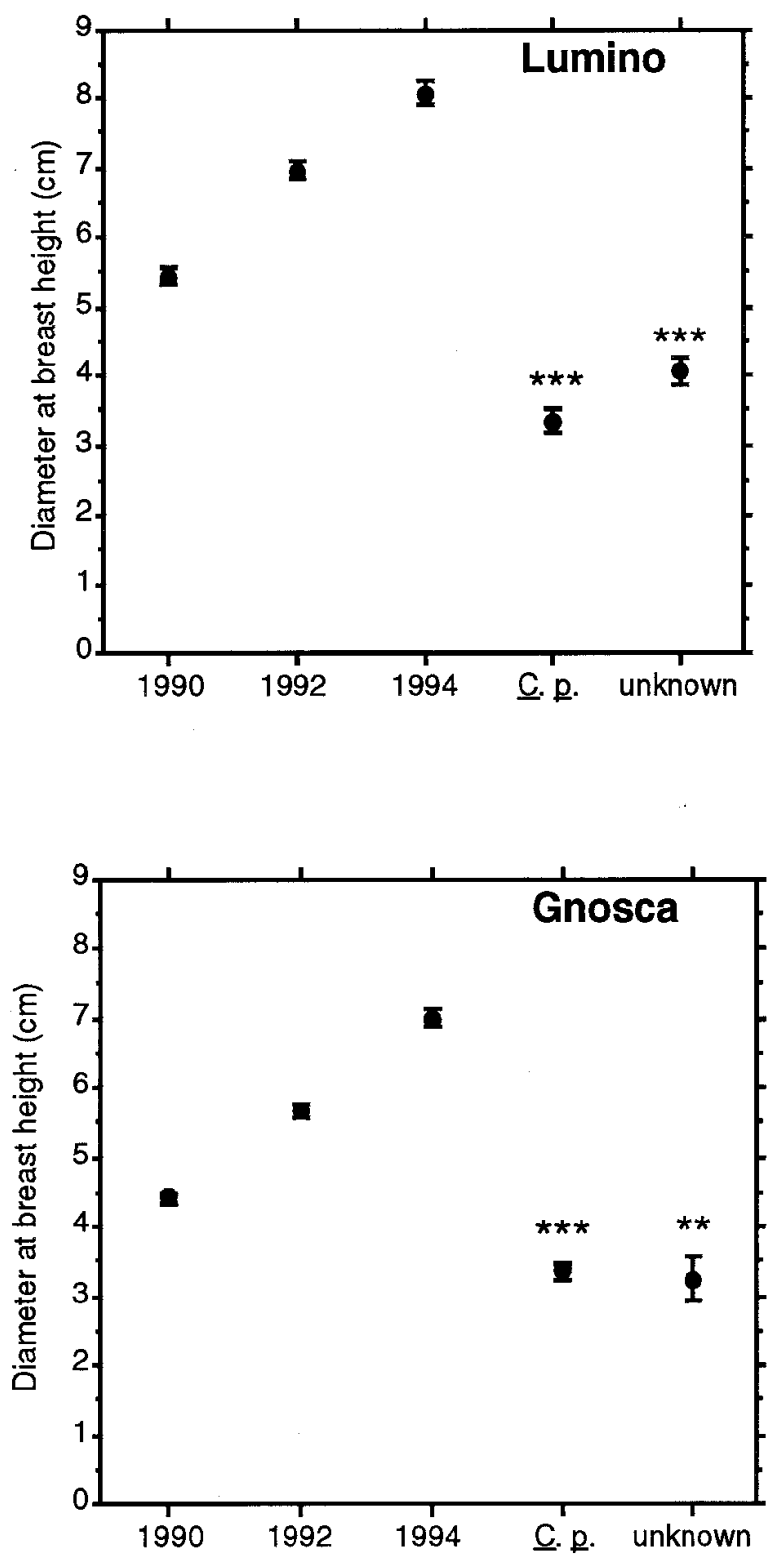

Fig. 5. Diameter of Castanea sativa at breast height. Mean diameter ( \pm standard error) of all living chestnut sprouts in Lumino and Gnosca in 1990, 1992, and 1994 and the diameter (in 1990) of the chestnut sprouts that died because of Cryphonectria parasitica cankers or for unknown reasons between 1990 and 1994 ( $^{* *}=P \leq 0.01 ; * * *=P \leq 0.001$, Student's twotailed $t$ test). white, hypovirulent strains. Our field studies produced evidence for a role of white $C$. parasitica isolates in the observed decrease of canker growth for a number of reasons. First, cankers that yielded white isolates in the beginning of the study grew slower than cankers yielding orange isolates. Second, cankers with white isolates killed considerably fewer sprouts than cankers with orange isolates. Third, cankers without growth for 2 subsequent years (1990 to 1992) frequently yielded more white than orange isolates. Finally, the majority of the cankers (80\% in Lumino; $66 \%$ in Gnosca) yielded white isolates at least once during the 4year observation period. However, the reduction in canker growth observed in the 4 years was not clearly linked to an increase of white isolates. When the ratios of orange to white isolates between years were compared, no clear picture was obtained. These results might be explained by the presence of both orange and white isolates in $C$. parasitica cankers, probably as a result of incomplete spread of the hypovirus (47) or multiple infections (33) within single cankers. About $30 \%$ of the cankers in both plots yielded isolates of a different VCG when resampled after 4 years (data not shown). For future studies, it will be necessary to increase the sample size per canker to get a more representative picture of the population structure in a single canker.

Chestnut blight development was fast at both Swiss investigation sites. In only 4 years, the portion of cankered sprouts rose from $37 \%$ to about $60 \%$. The actual increase of blight incidence was even higher, since only cankers up to $2 \mathrm{~m}$ above ground level were registered. The high disease incidence at the two study sites is not exceptional. Within 2 years, an increase of blighted sprouts from 39 to $61 \%$ was observed in Italy (50), where the same chestnut species and a similar fungal population are present. A survey in the Ticino showed that more than $50 \%$ of the chestnut trees were cankered (19). The blight incidence observed in our study is comparable with the incidence reported in Switzerland at the beginning of the epidemic. An increase of blighted sprouts from 20 to $40 \%$ was observed between 1958 and 1960 (46).

As shown in our study, chestnut blight killed $15 \%$ of the sprouts within 4 years. These sprouts, however, were among the thinnest sprouts. An additional $20 \%$ of thin sprouts were killed merely by competition among chestnut stems within a sprout cluster. In Europe, competition is a major factor of mortality in dense sprout coppices. Pagès and Cabanettes (42) investigated the competition among young European chestnuts in coppices. They found up to $50 \%$ dead sprouts per year in 1- to 2-year-old chestnut stands. Even in 10-year-old coppices, competition caused the death of $10 \%$ of the chestnuts per year. Pagès and Cabanettes (42) do not refer to chestnut blight. Both $C$. parasitica and competition seem to act as natural selection factors. They thin the dense coppice stands by eliminating the weak individuals in the sprout clusters. However, for every stump, several living sprouts remain. In contrast, in the United States, Griffin et al. (28) reported that only 16 to $73 \%$ of American chestnut (C. dentata) stumps in 10-year-old coppices had one or several living sprouts. Most of the young American chestnut sprouts were killed within 1 to 2 years after being blighted by $C$. parasitica $(26,40)$. Surviving chestnut sprouts were smaller than dead sprouts after blight attack in American clear-cuts (27).

TABLE 9. Mean vertical growth rates of all new Cryphonectria parasitica cankers in Lumino and Gnosca that developed on Castanea sativa between 1990 and 1994

\begin{tabular}{lrcccc}
\hline \multirow{2}{*}{$\begin{array}{l}\text { New } \\
\text { cankers in }\end{array}$} & & \multicolumn{4}{c}{ Mean canker growth $(\mathrm{cm})$ in the year } \\
\cline { 4 - 7 } & $N$ & 1991 & 1992 & 1993 & 1994 \\
\hline $1990-1991$ & 187 & $10.7 \pm 0.9^{\mathrm{a}}$ & $3.6 \pm 0.9$ & $2.5 \pm 0.8$ & $1.0 \pm 0.4$ \\
$1991-1992$ & 71 & $\ldots$ & $11.2 \pm 1.6^{\mathrm{a}}$ & $3.8 \pm 1.2$ & $1.5 \pm 0.7$ \\
$1992-1993$ & 88 & $\ldots$ & $\ldots$ & $12.5 \pm 1.6^{\mathrm{a}}$ & $3.9 \pm 1.4$ \\
$1993-1994$ & 52 & $\ldots$ & $\ldots$ & $\ldots$ & $12.5 \pm 1.9^{\mathrm{a}}$ \\
\hline
\end{tabular}

${ }^{a}$ Length of the cankers in the first year (August) of observation. 
The scarce literature from the beginning of the European chestnut epidemic indicates that the canker growth rate was high and continuous. For Italy, an average growth rate of $16 \mathrm{~cm} /$ year was reported (11). Schüepp (46) determined an average growth rate of more than $14 \mathrm{~cm}$ per year in southern Switzerland. In our plots, only newly established cankers showed a comparable growth rate in the first year. After that, the growth rate gradually diminished to about $1 \mathrm{~cm}$ within the next 3 years. This reduction in canker growth may be explained, for the most part, by the action of hypovirulence as discussed above.

A number of cankers in both plots that yielded only orange isolates over 4 years did not kill their host, suggesting that hypovirulence is not the only factor responsible for the reduction of the disease severity. It may be postulated that some orange $C$. parasitica strains have a reduced virulence or the sprouts have some resistance against the blight. Bazzigher (12) found a positive correlation between stem diameter of Castanea sativa and the portion of healed trees following artificial inoculations. Similarly, in this study, sprouts succumbing to $C$. parasitica were thinner than surviving sprouts. Bazzigher (12) attributed his observations to ontogenetic changes in predisposition of Castanea sativa. Our results did not reveal a reduction in mortality within the observation period (sprout age of 6 to 10 years). An age-dependent mortality rate for this period can, thus, be ruled out. Similarly, canker growth rates were not dependent on sprout age or sprout diameter. In their first year, the cankers always grew fast. This initial canker expansion probably causes severe damage only to thin sprouts, which does not allow recovery by hypovirulence or by a resistance response.

The decline of the European chestnut blight epidemic is still not thoroughly explained. Beside hypovirulence, the lower blight susceptibility of Castanea sativa compared with Castanea dentata seems to be an important factor (26). The mortality of European chestnuts may be delayed, which allows hypovirulence more time to infect and subsequently convert virulent $C$. parasitica cankers. A comprehensive study of the blight susceptibility of the two species, however, is still lacking. More experimental evidence is also needed to determine the relative contribution of hypovirulence and host resistance for the recovery of the European chestnuts.

\section{ACKNOWLEDGMENTS}

We thank H. Blauenstein, H. Cattelan, M. Conedera, P. Lawrenz, and the Forest Service of Canton Ticino for technical assistance. We also thank M. G. Milgroom for performing the spatial pattern analyses and O. Holdenrieder for helpful discussions.

\section{LITERATURE CITED}

1. Anagnostakis, S. L. 1977. Vegetative incompatibility in Endothia parasitica. Exp. Mycol. 1:306-316.

2. Anagnostakis, S. L. 1981. A stable heterokaryon of Endothia parasitica. Mycologia 73:570-576.

3. Anagnostakis, S. L. 1987. Chestnut blight: The classical problem of an introduced pathogen. Mycologia 79:23-37.

4. Anagnostakis, S. L. 1988. Cryphonectria parasitica, cause of chestnut blight. Adv. Plant Pathol. 6:123-136.

5. Anagnostakis, S. L. 1990. Improved chestnut tree condition maintained in two Connecticut plots after treatments with hypovirulent strains of the chestnut blight fungus. For. Sci. 36:113-124.

6. Anagnostakis, S. L. 1992. Diversity within populations of fungal pathogens on perennial parts of perennial plants. Pages 183-192 in: The Fungal Community. 2nd ed. G. C. Carrol and D. T. Wicklow, eds. Marcel Dekker, New York.

7. Anagnostakis, S. L., and Day, P. R. 1979. Hypovirulence conversion in Endothia parasitica. Phytopathology 69:1226-1229.

8. Anagnostakis, S. L., Hau, B., and Kranz, J. 1986. Diversity of vegetative compatibility groups of Cryphonectria parasitica in Connecticut and Europe. Plant Dis. 70:536-538.

9. Anagnostakis, S. L., and Kranz, J. 1987. Population dynamics of Cryphonectria parasitica in a mixed-hardwood forest in Connecticut. Phytopathology 77:751-754.
10. Anagnostakis, S. L., and Waggoner, P. E. 1981. Hypovirulence, vegetative incompatibility, and the growth of cankers of chestnut blight. Phytopathology 71:1198-1202.

11. Baldacci, E., and Picco, D. 1947. Il cancro del castagno. Humus 3:6-8.

12. Bazzigher, G. 1981. Selection of blight-resistant chestnut trees in Switzerland. Eur. J. For. Pathol. 11:199-207.

13. Bazzigher, G., Kanzler, E., and Kübler, T. 1981. Irreversible Pathogenitätsverminderung bei Endothia parasitica durch übertragbare Hypovirulenz. Eur. J. For. Pathol. 11:358-369.

14. Bissegger, M. 1993. Populationsstruktur und -dynamik von Cryphonectria (Endothia) parasitica in Schweizer Edelkastanienwäldern (Castanea sativa). Ph.D. dissertation. ETH, Zürich.

15. Bissegger, M., and Heiniger, U. 1994. Population structure of Cryphonectria parasitica in Swiss chestnut stands. Pages 143-147 in: Proc. Int. Chestnut Conf. M. L. Double and W. L. MacDonald, eds. West Virginia University Press, Morgantown, WV.

16. Bissegger, M., and Milgroom, M. G. 1994. Outcrossing rates of Cryphonectria parasitica in European chestnut (Castanea sativa) forests. (Abstr.) Phytopathology 84:1074.

17. Chen, B., Choi, G. H., and Nuss, D. L. 1993. Mitotic stability and nuclear inheritance of integrated viral cDNA in engineered hypovirulent strains of the chestnut blight fungus. EMBO (Eur. Mol. Biol. Organ.) J. 12:2991-2998

18. Choi, G. H., and Nuss, D. L. 1992. Hypovirulence of chestnut blight fungus conferred by an infectious viral cDNA. Science 257:800-803.

19. Conedera, M. 1991. La situazione del cancro del castagno (Cryphonectria (Endothia) parasitica (Murr.) Barr) al Sud delle Alpi (Svizzera meridionale). Schweiz. Z. Forstwes. 142:283-298.

20. Day, P. R., Dodds, J. A., Elliston, J. E., Jaynes, R. A., and Anagnostakis, S. L. 1977. Double-stranded RNA in Endothia parasitica. Phytopathology 67:1393-1396.

21. Elliston, J. E. 1985. Characteristics of dsRNA-free and dsRNA-containing strains of Endothia parasitica in relation to hypovirulence. Phytopathology 75:151-158.

22. Fulbright, D. W., Weidlich, W. H., Haufler, K. Z., Thomas, C. S., and Paul, C. P. 1983. Chestnut blight and recovering American chestnut trees in Michigan. Can. J. Bot. 61:3164-3171.

23. Garbelotto, M., Frigimelica, G., and Mutto-Accordi, S. 1992. Vegetative compatibility and conversion to hypovirulence among isolates of Cryphonectria parasitica from northern Italy. Eur. J. For. Pathol. 22:337348 .

24. Grente, J. 1965. Les formes hypovirulentes d'Endothia parasitica et les espoirs de lutte contre le chancre du châtaignier. C. R. Acad. Agric. Fr. 51:1033-1037.

25. Grente, J. 1981. Les variants hypovirulents de l'Endothia parasitica et la lutte biologique contre le chancre du châtaignier. Ph.D. thesis. Université de Bretagne Occidentale, Brest, France.

26. Griffin, G. J. 1986. Chestnut blight and its control. Hort. Rev. 8:291-336.

27. Griffin, G. J. 1989. Incidence of chestnut blight and survival of American chestnut in forest clearcut and neighboring understory sites. Plant Dis. 73:123-127.

28. Griffin, G. J., Smith, H. C., Dietz, A., and Elkins, J. R. 1991. Importance of hardwood competition to American chestnut survival, growth, and blight development in forest clearcuts. Can. J. Bot. 69:1804-1809.

29. Hebard, F. V. 1982. Biology of virulent and hypovirulent Endothia parasitica on American chestnut (Castanea dentata). Ph.D. dissertation. Virginia Polytechnic Institute and State University, Blacksburg, VA.

30. Heiniger, U., and Rigling, D. 1994. Biological control of chestnut blight in Europe. Annu. Rev. Phytopathol. 32:581-599.

31. Hillman, B. I., Fulbright, D. W., Nuss, D. L., and van Alfen, N. K. 1995. Hypoviridae. Pages 261-264 in: Rep. Int. Committee Taxon. Viruses, 6th. F. A. Murphy, C. M. Fauquet, D. H. L. Bishop, S. A. Ghabrial, A. W. Jarvis, G. P. Martelli, M. A. Mayo, and M. D. Summer, eds. SpringerVerlag, New York.

32. Hillman, B. I., Shapira, R., and Nuss, D. L. 1990. Hypovirulence-associated suppression of host functions in Cryphonectria parasitica can be partially relieved by high light intensity. Phytopathology 80:950-956.

33. Kuhlman, E. G., and Bhattacharyya, H. 1984. Vegetative compatibility and hypovirulence conversion among naturally occurring isolates of Cryphonectria parasitica. Phytopathology 74:659-664.

34. Kuhlman, E. G., Bhattacharyya, H., Nash, B. L., Double, M. L., and MacDonald, W. L. 1984. Identifying hypovirulent isolates of Cryphonectria parasitica with broad conversion capacity. Phytopathology 74:676-682.

35. Liu, Y.-C., and Milgroom, M. G. 1996. Correlation between hypovirus transmission and the number of vegetative incompatibility (vic) genes different among isolates from a natural population of Cryphonectria parasitica. Phytopathology 86:79-86.

36. MacDonald, W. L., and Double, M. L. 1978. Frequency of vegetative 
compatibility types of Endothia parasitica in two areas of West Virginia. Pages 103-105 in: Proc. Am. Chestnut Symp. W. L. MacDonald, F. C. Cech, J. Luchok, and C. Smith, eds. West Virginia University Press, Morgantown, WV

37. MacDonald, W. L., and Fulbright, D. W. 1991. Biological control of chestnut blight: Use and limitations of transmissible hypovirulence. Plant Dis. 75:656-661.

38. Milgroom, M. G. 1995. Population biology of the chestnut blight fungus Cryphonectria parasitica. Can. J. Bot. 73(suppl. 1):S311-S319.

39. Milgroom, M. G., Lipari, S. E., Ennos, R. A., and Liu, Y. C. 1993. Estimation of the outcrossing rate in the chestnut blight fungus, Cryphonectria parasitica. Heredity 70:385-392.

40. Milgroom, M. G., MacDonald, W. L., and Double, M. L. 1991. Spatial pattern analysis of vegetative compatibility groups in the chestnut blight fungus, Cryphonectria parasitica. Can. J. Bot. 69:1407-1413.

41. Nuss, D. L. 1992. Biological control of chestnut blight: An example of virus-mediated attenuation of fungal pathogenesis. Microbiol. Rev. 56:561-576.

42. Pagès, L., and Cabanettes, A. 1993. Évolution de la densité des rejets et structure des peuplements dans les taillis de châtaignier. Acta Oecol. 14:823-838.
43. Rigling, D. 1995. Isolation and characterization of Cryphonectria parasitica mutants that mimic a specific effect of hypovirulence-associated dsRNA on laccase activity. Can. J. Bot. 73:1655-1661.

44. Rigling, D., Heiniger, U., and Hohl, H. R. 1989. Reduction of laccase activity in dsRNA-containing hypovirulent strains of Cryphonectria (Endothia) parasitica. Phytopathology 79:219-223.

45. Rizwana, R., and Powell, W. A. 1992. Ultraviolet light-induced instability of vegetative compatibility groups of Cryphonectria parasitica. Phytopathology 82:1206-1211.

46. Schüepp, H. 1961. Erhebungen über das Kastaniensterben im Kanton Tessin. Mitt. EAFV 37:159-169.

47. Shain, L., and Miller, J. B. 1992. Movement of cytoplasmic hypovirulence agents in chestnut blight cankers. Can. J. Bot. 70:557-561.

48. van Alfen, N. K., Jaynes, R. A., Anagnostakis, S. L., and Day, P. R. 1975. Chestnut blight: Biological control by transmissible hypovirulence in Endothia parasitica. Science 189:890-891.

49. Vrot, F., and Grente, J. 1985. Le chancre de l'écorce du châtaignier. Phytoma Déf. Cult. Mars:35-37.

50. Zechini D’Aulerio, A., and Zambonelli, A. 1987. Virulenza ed ipovirulenza di Endothia parasitica in un castagneto da frutto. Monti Boschi 2:20-24. 